\title{
ESCO1/2's roles in chromosome structure and interphase chromatin organization
}

\author{
Ryotaro Kawasumi, ${ }^{1,4}$ Takuya Abe, ${ }^{1,2,4}$ Hiroshi Arakawa, ${ }^{1}$ Massimiliano Garre, ${ }^{1}$ Kouji Hirota, ${ }^{2}$ \\ and Dana Branzei ${ }^{1,3}$ \\ ${ }^{1}$ The FIRC (Italian Foundation for Cancer Research) Institute of Molecular Oncology (IFOM), 20139 Milan, Italy; ${ }^{2}$ Department of \\ Chemistry, Graduate School of Science and Engineering, Tokyo Metropolitan University, Hachioji-shi, Tokyo 192-0397, Japan; \\ ${ }^{3}$ Istituto di Genetica Molecolare, Consiglio Nazionale delle Ricerche (IGM-CNR), 27100 Pavia, Italy
}

ESCO1/2 acetyltransferases mediating SMC3 acetylation and sister chromatid cohesion (SCC) are differentially required for genome integrity and development. Here we established chicken DT40 cell lines with mutations in ESCO1/2, SMC3 acetylation, and the cohesin remover WAPL. Both ESCO1 and ESCO2 promoted SCC, while ESCO2 was additionally and specifically required for proliferation and centromere integrity. ESCO1 overexpression fully suppressed the slow proliferation and centromeric separation phenotypes of esco 2 cells but only partly suppressed its chromosome arm SCC defects. Concomitant inactivation of ESCO1 and ESCO2 caused lethality owing to compromised mitotic chromosome segregation. Neither wapl nor acetyl-mimicking smc3-QQ mutations rescued esco1 esco2 lethality. Notably, esco1 esco2 wapl conditional mutants showed very severe proliferation defects associated with catastrophic mitoses and also abnormal interphase chromatin organization patterns. The results indicate that cohesion establishment by vertebrate ESCO1/2 is linked to interphase chromatin architecture formation, a newly identified function of cohesin acetyltransferases that is both fundamentally and medically relevant.

[Keywords: cohesin acetyltransferases; ESCO1/2; centromere; chromatin territories; chromosome segregation; sister chromatid cohesion]

Supplemental material is available for this article.

Received August 14, 2017; revised version accepted November 9, 2017.

Sister chromatid cohesion (SCC) is mediated by the cohesin complex, which minimally consists of SMC3, SMC1, RAD21 (also known as Mcd1 or Scc1), and SA1/SA2 (also known as Scc3 or Irr1). Cohesin associates transiently with other factors, including PDS5A/B, Sororin, and WAPL, which play roles in cohesion establishment, maintenance, and dissolution (Peters and Nishiyama 2012). It is known that cohesin loading on chromatin per se is not sufficient to promote SCC. During S phase, budding yeast Ecol acetyltransferase converts cohesin into a cohesive form (Skibbens et al. 1999; Ivanov et al. 2002). One of the key substrates of budding yeast Ecol is Smc3 (Rolef Ben-Shahar et al. 2008; Unal et al. 2008; Zhang et al. 2008; Onn et al. 2009), which is acetylated at two evolutionarily conserved lysine residues: K112 and K113. This mechanism is conserved in mammalian cells in which corresponding K105 and K106 residues of SMC3 are acetylated (Zhang et al. 2008). The essential function of Ecol in Saccharomyces cerevisiae is linked to Smc3 acetylation and its role in counteracting the anti-cohesion factor WAPL (also known as Rad61 or Wpl1). This concept

\footnotetext{
${ }^{4}$ These authors equally contributed to this work.

Corresponding authors: dana.branzei@ifom.eu, 0330abe@tmu.ac.jp

Article published online ahead of print. Article and publication date are online at http://www.genesdev.org/cgi/doi/10.1101/gad.306084.117. Freely available online through the Genes \& Development Open Access option.
}

emerged from findings that in S. cerevisiae, deletion of WPL1 or acetyl-mimicking mutants of Smc3 (K113N or $\mathrm{K} 113 \mathrm{Q}$ ) rescues the lethality of eco1 $\Delta$ cells (Rolef Ben-Shahar et al. 2008; Unal et al. 2008; Peters and Bhaskara 2009; Rowland et al. 2009; Sutani et al. 2009). However, additional studies showing that eco1 $\Delta$ wpl1 $\Delta$ and acetyl-mimicking mutants of cohesin fail to restore SCC (Guacci and Koshland 2012) challenge the dogma that Ecol's essential function is strictly related to cohesion. Moreover, in budding yeast, the cohesion defects of replisome-associated factors that promote cohesion and facilitate cohesin acetylation are not suppressed by removing Wapl (Borges et al. 2013). Thus, even in budding yeast, the interplay between Ecol, cohesin acetylation, and WAPL in cohesion and proliferation is likely more complex than initially thought and needs careful consideration (Rudra and Skibbens 2013).

In vertebrates, the roles of ESCO1/2 and cohesin acetylation in proliferation and specific chromosome structural processes have not yet been formerly addressed. Moreover, it is important to note several differences between frequently used model systems. First, budding and

(C) 2017 Kawasumi et al. This article, published in Genes \& Development, is available under a Creative Commons License (Attribution-NonCommercial 4.0 International), as described at http://creativecommons.org/licenses/by-nc/4.0/. 
fission yeast have only one cohesin acetyltransferase, whereas vertebrate cells have two: ESCO1 and ESCO2. The human ESCO1 and ESCO2 show strikingly distinct patterns of chromatin association in somatic cells, directed by their divergent $\mathrm{N}$-terminal motifs that are not conserved in budding yeast Ecol (Hou and Zou 2005; Rahman et al. 2015). Second, budding yeast is an exception regarding the mechanisms by which cohesin is removed and recycled from the chromosomes. In S. cerevisiae, cohesin removal depends entirely on cleavage of $\operatorname{Scc} 1 / \operatorname{Rad} 21$ at anaphase by a protein called separase, whereas, in vertebrates, WAPL removes cohesin from chromosome arms during prophase as intact rings, and only a small pool of cohesin at centromeres remains to be cleaved by the protease separase at anaphase (Peters and Nishiyama 2012; Tedeschi et al. 2013). Third, cohesin and ESCO1/2 play roles in controlling gene expression (Rahman et al. 2015). These mechanisms are potentially different from budding yeast in which other coregulators of the cohesin function in transcription, such as CTCF, are missing (Peters and Nishiyama 2012). Finally, haploid genomes such as those of budding and fission yeast may have different regulations compared with diploid ones. Therefore, analysis of the phenotypes associated with defective or constitutive SMC3 acetylation and of the ones caused by singular or combinatorial ESCO1/2 dysfunction in somatic cells is a timely topic with the potential to reveal novel aspects of chromosome structure and SCC regulation in the vertebrate genome.

ESCO1's and ESCO2's functions are important to study also because of their implication in genome integrity and development. ESCO1 mutations and overexpression appear critical in a variety of tumors. ESCO1 mutations were correlated with endometrial cancers (Price et al. 2014), and ESCO1 is classified as a susceptibility DNA repair gene implicated in a common somatic fusion in prostate cancers (Luedeke et al. 2009). ESCO1 is also amplified in many cancers (http://www.cbioportal.org), and its overexpression in bladder cancers is now an independent prognostic biomarker for patients with bladder cancer (Zhang et al. 2016). On the other hand, mutations in human ESCO2 cause a hereditary developmental disease called Roberts syndrome (RBS), classified as cohesinopathy (Vega et al. 2005), and ESCO2 deletions are common in cancers (http://www.cbioportal.org). ESCO2 knockout mice are embryonic-lethal, and ESCO2-deleted mouse embryonic fibroblasts (MEFs) show severe chromosome segregation defects (Whelan et al. 2012). These findings indicate that ESCO2's functions during proliferation are not physiologically compensated for by ESCO1. However, except for a broader role of ESCO1 compared with ESCO2 in repressing transcription in somatic cells (Rahman et al. 2015), the functions commonly or differentially performed by ESCO1 and ESCO2 during somatic proliferation remain largely unknown.

It was shown recently that the acetylation of the SMC3 subunit of cohesin at conserved lysine residues K105 and K106 by ESCO1/ESCO2 reduces cohesin mobility and promotes stable binding of Sororin to cohesin, presumably to enhance SCC (Ladurner et al. 2016). Both ESCO1 and
ESCO2 can acetylate SMC3 at K105 and K106, although ESCO1 can acetylate cohesin independently of DNA replication (Minamino et al. 2015). The roles of vertebrate ESCO1, ESCO2, and SMC3 acetylation and their functional interplay in different processes related to proliferation have not been addressed to date, one of the reasons likely being related to the technical challenges of conditionally and concomitantly mutating/inactivating multiple genes in vertebrate cells.

Here we used genetically amenable chicken DT40 cells to establish knockout and knock-in of several cohesin regulatory genes and mutant alleles. In addition, we adapted the auxin-degron system (Nishimura et al. 2009) to DT40 cells to generate efficient conditional depletion of factors. Our results unveil an important role for ESCO2 in proliferation, at centromeres, and in the functional interaction between ESCO1 and ESCO2 acetyltransferases with regard to proliferation and the establishment/maintenance of centromeric sister chromatid proximity. We found that mimicking SMC3 acetylation at K105 and K106 does not bypass the essential function performed jointly by ESCO1 and ESCO2 or the function of ESCO1 in promoting chromosome arm SCC. Cohesin is stabilized in esco1 esco2 cells by conditional inactivation of WAPL, but the triple conditional mutant esco1 esco2 wapl has very severe proliferation defects and abnormal interphase chromatin territories. Together, our findings reveal a functional interaction between ESCO1 and ESCO2 in supporting centromere integrity and chromosome segregation via mechanisms that do not singularly rely on cohesin acetylation at K105 and K106 and identify a role of vertebrate ESCO1/2 in interphase chromosome territory organization.

\section{Results}

ESCO2, but not ESCO1, is critical for proliferation and centromere integrity

We previously established ESCO2 knockout cell lines in DT40 cells (Abe et al. 2016). To generate DT40 cell lines deleted for ESCO1, we designed knockout constructs that delete most of the catalytic domain of ESCO1 (Fig. 1A). The chicken ESCO1 gene is located on chromosome 2 , which is present in three copies in DT40 cells. We verified the correct establishment of $E S C O 1^{-/-/-}$strains by RT-PCR (Fig. 1B). In contrast to $E S C O 2^{-/-}$cells that are characterized by severe growth defects (see also Abe et al. 2016), the proliferation rate of ESCO1 $1^{-/-/-}$mutants was similar to that of wild-type cells (Fig. 1C).

Both human ESCO1 and ESCO2 contribute to SCC and have acetyltransferase activities in vitro (Hou and Zou 2005). However, in human cells, ESCO1 is more important for SMC3 acetylation at K105 and K106 residues, judging from the effects of inactivating ESCO1 and ESCO2 with siRNA (Zhang et al. 2008), possibly due to the fact that ESCO1-mediated acetylation occurs also outside of the DNA replication window (Minamino et al. 2015). To address the situation in DT40, we next monitored acetylated SMC3 (Ac-SMC3) versus total SMC3 in 

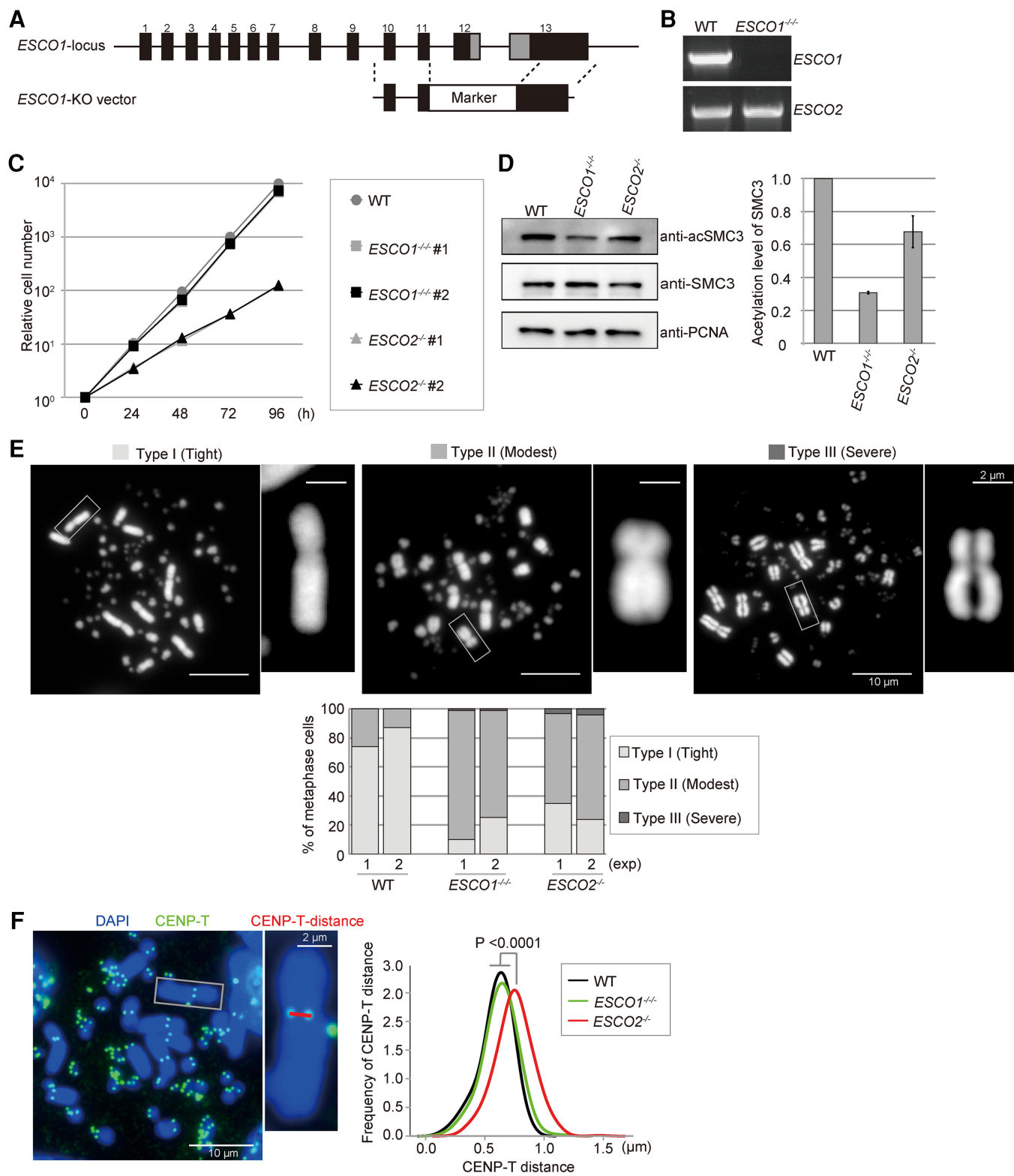

Figure 1. ESCO2, but not ESCO1, is critical for proliferation and centromere integrity. (A) Schematic representation of the ESCO1 gene locus and gene targeting knockout construct. (Closed boxes) Exons; (Marker) drug resistance genes; (gray box) the sequence encoding the acetyltransferase domain of ESCO1. (B) RT-PCR. Disruption of the ESCO1 gene was ultimately verified by RT-PCR using an ESCO1-specific primer set. The ESCO2 gene was used as a control. $(C)$ Growth curves of the indicated cell lines. Cells $\left(1 \times 10^{5}\right)$ of the indicated genotypes were inoculated in $1 \mathrm{~mL}$ of medium, counted, and passaged every $24 \mathrm{~h}$. (D) The SMC3-K105, K106 acetylation level was measured. Whole-cell lysates were prepared from cells of the indicated genotypes. Acetylated SMC3, SMC3, and PCNA (loading control) were detected by Western blotting. (E) Chromosomes from metaphase spreads were classified into three groups, and $>100$ metaphase cells were analyzed for each genotype. The results of two independent experiments are plotted. $(F)$ Metaphase spread samples were prepared by the cytospin method after incubation with $100 \mathrm{ng} / \mathrm{mL}$ colcemid for $1 \mathrm{~h}$. The distances between CENP-T signals were measured for $>400$ chromosomes. The same trend was confirmed from an independent biological experiment. $P$-values were calculated by Student's t-test.

the established esco1 and esco2 knockout cell lines. The Ac-SMC3 level was decreased in both mutants, but the decrease was more pronounced in ESCO1 ${ }^{-/-/-}$cells than in $\mathrm{ESCO}^{-/-}$cells (Fig. 1D).

We next examined metaphase spreads of $\mathrm{ESCO}^{-/-1-}$ and $\mathrm{ESCO}^{-/-}$cells to score for cohesion defects. As re- ported previously (Stephan et al. 2011; Era et al. 2012; Abe et al. 2016), a high fraction of metaphases in wildtype DT40 cells has well-cohered tight chromosomes (Fig. 1E, type I). Only 5\%-25\% of wild-type metaphase cells have chromosomes in which the arms are slightly apart, a phenotype scored as a chromosome arm 
SCC defect (Fig. 1E, type II). Notably, $70 \%-90 \%$ of ESCO1 $1^{-1-1-}$ and $E S C O 2^{-1-}$ metaphases showed chromosomes with arm cohesion defects of type II. Moreover, a small fraction of metaphases in esco1 and esco2 cells had also severe cohesion defects of type III, with chromosomes separated also at centromeres (Fig. 1E). Thus, both ESCO1 and ESCO2 significantly contribute to chromosome arm SCC in nonredundant ways, consistent with previous observations in human cells (Hou and Zou 2005). Importantly, however, the proliferation defect of ESCO2 $^{-/-}$(Fig. 1C) does not correlate with SMC3 acetylation or chromosome arm SCC defects, which were more pronounced in $E S C O 1^{-/-/-}$cells than in $\mathrm{ESCO}^{-/-}$cells (Fig. 1D,E).

Next, we analyzed whether centromeric sister chromatid separation is more severe in $\mathrm{ESCO}^{-/-}$cells than in ESCO1-/-/- cells. To do so, we measured the distance between CENP-T signals marking the sister centromeres in metaphase spread cells (Fig. 1F; see Abe et al. 2016). The average distance between CENP-T signals was very similar in wild-type and ESCO1 $1^{-1-/-}$ cells but was increased in ESCO $2^{-/-}$mutants (Fig. $1 \mathrm{~F}$ ), suggesting that ESCO2 specifically engenders centromeric sister chromatid proximity.

Previously, we found synthetic lethality between a hypomorphic ESCO2 mutant that mimics a relatively common mutation, W539G, found in RBS patients $\left(E S C O 2^{-/ W 615 G}\right)$ and conditional inactivation of $D D X 11$, which is mutated in another cohesinopathy known as the Warsaw breakage syndrome (see below; Abe et al. 2016). The synthetic lethality of ESCO2 $2^{-/ W 615 G} d d x 11$ was associated with inner centromere dysfunction and chromosome missegregation (Abe et al. 2016). We addressed whether, similarly to the esco2-W615G mutation, which does not have proliferation and centromere defects on its own (Abe et al. 2016), ESCO1 ablation may affect centromere function in a subtle way that could be exposed when DDX11 was concomitantly inactivated. However, differently from the esco2-W615G mutation, the ESCO1 $1^{-1-1-}$ mutation did not severely aggravate the proliferation of $D D X 11^{-/-}$cells (Supplemental Fig. S1A), substantiating the notion of different physiological contributions of ESCO1 and ESCO2 to centromeric structure/ cohesion.

Altogether, the results show that both ESCO1 and ESCO2 contribute to chromosome arm SCC, but ESCO2 is key for centromere function. Moreover, the correlation between centromeric sister chromatid separation and proliferation defects in $E S C O 2^{-/-}$cells indicates that the former may underlie the latter, a hypothesis further corroborated by this study (see below).

\section{Combined deficiency in ESCO1 and ESCO2 causes lethality}

To further examine the genetic relationship between ESCO1 and ESCO2, we generated ESCO1-/-/ESCO2 $2^{-/ 3 A I D 6 F l a g}$ conditional cells in which the ESCO2 protein can be down-regulated by addition of Auxin. To establish these cell lines, we followed the procedure described in Figure 2A. Briefly, we expelled the markers in
ESCO1-/-/- cells by the action of tamoxifen-induced Cre, disrupted the first allele of the ESCO2 gene, added a 3AID-6Flag tag to the second allele of ESCO2 (Kobayashi et al. 2015), and expressed TIR1-9myc, which is required for the degradation of AID-tagged substrates. After Auxin addition, the ESCO2-3AID-6Flag protein disappeared within $3 \mathrm{~h}$ (Fig. 2B). The levels of Ac-SMC3, already strongly impaired in ESCO1 $1^{-/-1-}$ ESCO2 $2^{-/ 3 A I D 6 F l a g}$ due to the absence of ESCO1 and only half levels of ESCO2, strongly declined $3 \mathrm{~h}$ after Auxin treatment (Fig. 2B). The proliferation of conditionally inactivated ESCO1-/-/ESCO2-/3AID6Flag (esco1 esco2) cells was severely impaired, with cells stopping to proliferate $12 \mathrm{~h}$ after Auxin addition (Fig. 2C). Notably, ESCO1 $1^{-/-/-}$ESCO2 $2^{-/ 3 A I D 6 F l a g}$ cells treated with Auxin showed a strong increase in metaphases exhibiting centromeric separation defects (type III), observed with only low frequency in $\mathrm{ESCO}^{-{ }^{--}}$and ESCO1 $1^{-/-/-}$cells (Fig. 2D). This increase in cells with separated centromeres was also accompanied by an increased percentage of anaphase cells with lagging chromosomes, marked by CENP-T signals lagging between the two separating DAPI-stained DNA bundles (Fig. 2E). Thus, ESCO1 and ESCO2 act jointly to support centromere function and proliferation, with ESCO2 being more important in this regard.

Proliferation and centromeric SCC defects of esco2 cells are compensated for by ESCO1 overexpression

Since $E S C O 2^{-/-}$cells have severe growth defects (Fig. 1C; Abe et al. 2016), we asked whether increasing the expression level of ESCO1 would compensate for ESCO2 loss. To address this possibility, we overexpressed ESCO1Flag in ESCO2--/ cells. The levels of ESCO1-Flag (expressed from the chicken $\beta$-actin promoter) in two selected $E S C O 2^{-/-}$clones were fivefold and 10-fold the endogenous levels of ESCO1 in wild-type and ESCO2 ${ }^{-1-}$ cells, respectively (Fig. 3A). These levels of ESCO1-Flag overexpression proportionally increased the levels of AcSMC3 in ESCO2 ${ }^{-/-}$cells above wild-type levels (Fig. $3 \mathrm{~B})$, and both fully suppressed the proliferation defects of $E S C O 2^{-/-}$cells (Fig. 3C, note overlap in the growth curves of the two tested $E S C O 2^{-/-}$clones overexpressing ESCO1). As the results in Figure 1 indicated similar trends in the proliferation and centromeric separation defects of $E_{S C O} 2^{-/-}$cells (Fig. 1C,F), we addressed whether ESCO1 overexpression also suppresses the centromeric separation defect of ESCO2 $2^{-1-}$ cells. This was indeed the case (Fig. 3D).

To test whether ESCO1 overexpression can also suppress the synthetic lethality between ESCO2 ${ }^{-/ W 615 G}$ and DDX11 shutoff (induced by addition of doxycycline [Dox] to ESCO2-/W615G cell lines expressing Tet-off$D D X 11)$, similarly to its effect in $E_{S C O 2^{-1-}}$ cells (Fig. 3C), we overexpressed ESCO1-Flag in ESCO2 $2^{-/ W 615 G}$ Tet-off-DDX11 cells. We selected two clones overexpressing ESCO1-Flag (Supplemental Fig. S1B) and used them for further assays. Notably, both the lethality and the high frequency of lagging chromosomes in ESCO2 $2^{-/ W 615 G}$ 
A

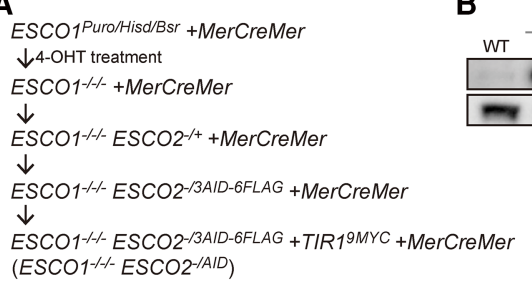

C

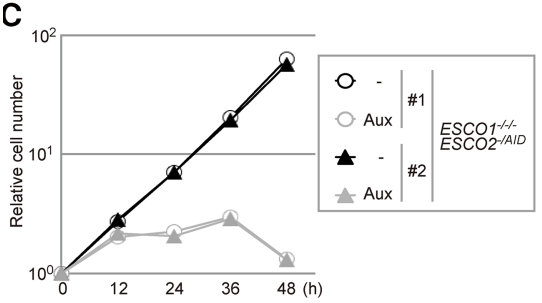

$\mathbf{E}$

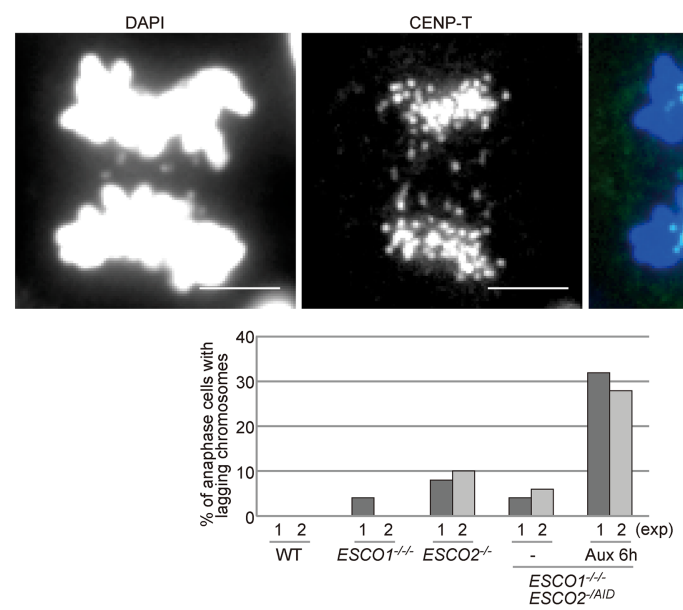

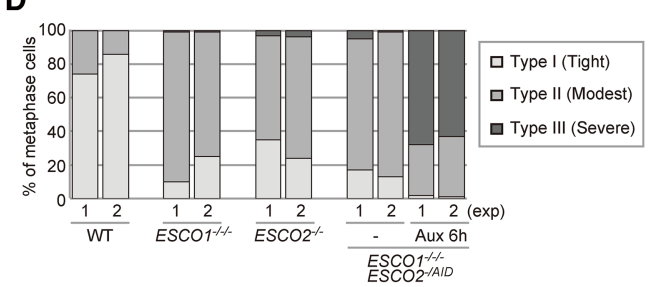
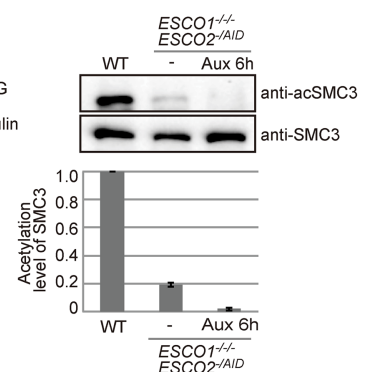

$-m$ anti-Tubulin

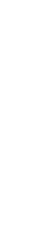

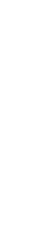

Merge

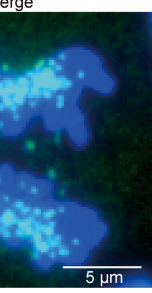

Figure 2. Combined deficiency in ESCO1 and ESCO2 causes lethality. $(A)$ Scheme of the conditional double mutant's establishment. $(B)$ Depletion of the ESCO2-3AID-6Flag protein and measurement of Ac-SMC3, SMC3, and a-tubulin (loading control) by immunoblotting. The results were confirmed with lysates from an independent biological experiment. $(C)$ Growth curves. Cells $\left(1 \times 10^{5}\right)$ of the indicated genotypes were inoculated in $1 \mathrm{~mL}$ of medium, counted, and passaged every $12 \mathrm{~h}$. Auxin (500 $\mu \mathrm{M}$ final) was added at time 0 as required. $(D)$ Chromosomes from metaphase spreads were analyzed as in Figure 1E. Auxin (500 $\mu \mathrm{M}$ final) was added $6 \mathrm{~h}$ before cell collection and analysis. (E) Lagging chromosomes in anaphase cells. At least 50 anaphase cells were analyzed for each experiment. The results of two independent experiments are plotted. Auxin $(500 \mu \mathrm{M}$ final) was added $6 \mathrm{~h}$ before cell collection.

$D D X 11^{-/-}$anaphase cells (Fig. 3E,F) were suppressed by ESCO1-Flag overexpression.

We next tested whether the effect of ESCO1 overexpression is specific to esco2 mutations (i.e., ESCO2 $2^{-1-}$ and $E S C O 2^{-/ W 615 G}$ ) or also bypasses the SCC defects caused by DDX11 dysfunction. Notably, ESCO1-Flag overexpression did not compensate for the SCC defects of $d d \times 11$ cells (Supplemental Fig. S1C,D). Thus, the cohesion defect of $D D X 11^{-/-}$cells is not associated with a robust defect in SMC3 acetylation (Abe et al. 2016) or rescued by genetic alterations that cause increased levels of AcSMC3.

The above results raised interest regarding whether high levels of ESCO1 can suppress specifically centromere-related phenotypes associated with ESCO2 dysfunction (Fig. 3C-F) or also compensate for arm SCC defects in esco2 mutants. ESCO1 overexpression only partly sup- pressed the chromosome arm cohesion defects of ESCO2 $^{-/-}$cells (Fig. 3G), and this may be caused by the restoration of centromeric sister chromatid proximity (see Fig. 3D). Altogether, the results indicate a functional interaction between ESCO1 and ESCO2 in supporting proliferation and centromere function and reveal that the proliferation and chromosome arm SCC functions of ESCO1/ 2 are at least partly uncoupled from each other and from cohesin acetylation.

\section{SMC3-K105, K106 acetylation is not singularly responsible for ESCO1's and ESCO2's roles in proliferation}

As SMC3 is the most studied and discussed target of ESCO1/2 (Minamino et al. 2015; Ladurner et al. 2016), we next sought to address the effects of defective or 
A

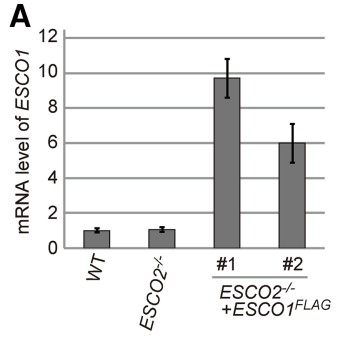

D
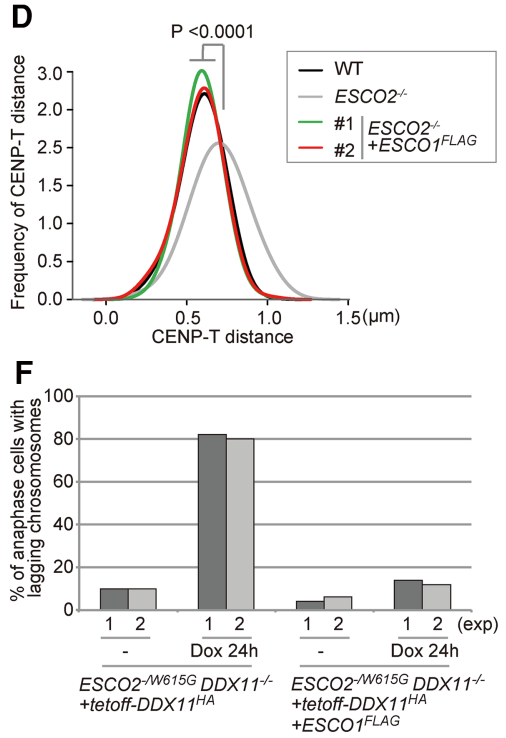

B

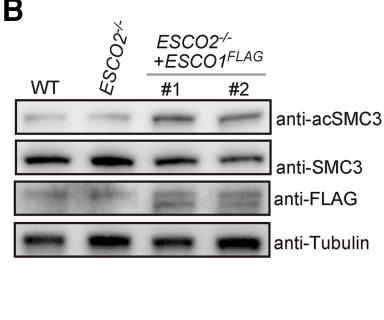

C

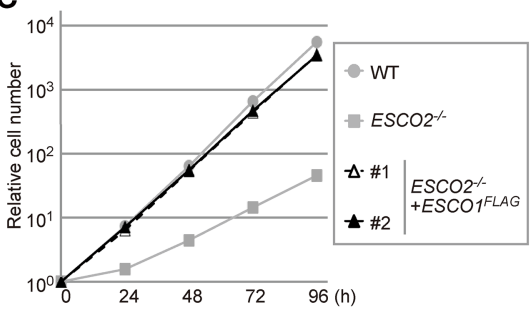

$\mathbf{E}$

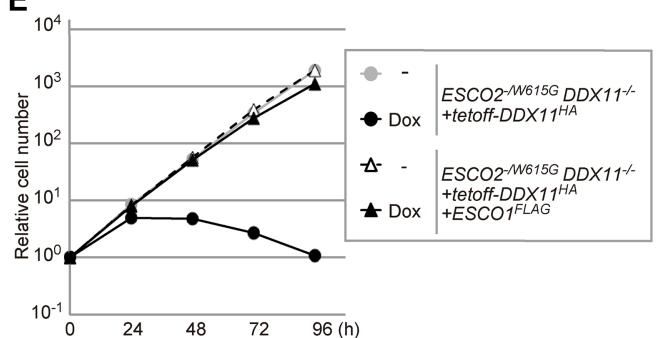

G

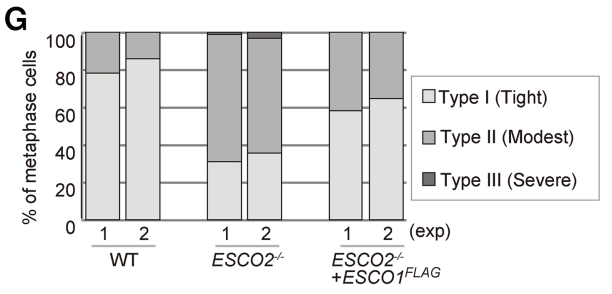

Figure 3. Proliferation and centromeric separation defects of esco2 cells are compensated for by ESCO1 overexpression. (A) ESCO1 mRNA levels were measured by quantitative PCR. (B) ESCO1-Flag protein level and the acetylation level of SMC3 were measured by Western blotting. $(C)$ Growth curves are as in Figure 1C. $(D)$ The distances between CENP-T signals were measured for $>400$ chromosomes, as in Figure 1F. (E) Growth curves are as in Figure 1C. Doxycycline (Dox; $1 \mu \mathrm{g} / \mathrm{mL}$ final) was added at time 0, when indicated. $(F)$ The measurement of lagging chromosomes in anaphase cells was as in Figure 2E. Dox was added $24 \mathrm{~h}$ before sample collection, as indicated. $(G)$ Chromosomes from metaphase spreads were analyzed for cohesion defects following the classification procedure outlined in Figure 1E.

constitutive acetylation of SMC3 at the conserved K105, K106 sites on proliferation and SCC. Acetyl-mimicking mutations of K105 and K106 sites of SMC3 reduce cohesin mobility (Ladurner et al. 2016), but whether SMC3 acetylation underlies the essential function of ESCO1 and ESCO2 in proliferation (see Fig. $2 \mathrm{C}$ ) and whether that essential function is linked to SCC are not known. To examine this issue, we introduced the $S M C 3^{K 105 \mathrm{Q} K 106 \mathrm{Q} /-}$

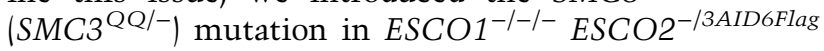
cell lines. To achieve this, we first knocked in the K105Q, K106Q (QQ) mutations on one allele of SMC3 (see Supplemental Fig. S2A for the SMC3 knock-in construct) in ESCO1 ${ }^{-1-1-}$ ESCO2 ${ }^{-/ 3 A I D 6 F l a g}$ cells (Fig. 4A). Next, we removed the marker cassette used for knock-in of $S M C 3-Q Q$ by inducing the Cre recombinase with tamoxifen (Fig. 4A; Supplemental Fig. S2A). Subsequently, we knocked out the second allele of $S M C 3$ using a vector that disrupts the first two exons of SMC3 (Supplemental Fig. S2A) to obtain ESCO1-1-/- ESCO2 $2^{-/ 3 A I D 6 F l a g}$ $S M C 3^{\mathrm{QQ} /-}$ cell lines (Fig. 4A). The mutations introduced in SMC3 were checked by cDNA sequencing (Supplemental Fig. S2B) and Western blotting, as the SMC3-QQ vari- ant is not recognized by the antibody against Ac-SMC3 (Supplemental Fig. S2C).

Notably, the smc3-QQ acetyl-mimicking mutation did not suppress the conditional lethality, SCC defects, or increased frequency of lagging chromosomes of esco1 esco2 cells (Fig. 4B-D). Thus, the essential function performed jointly by ESCO 1 and ESCO 2 in proliferation and chromosome segregation cannot be singularly attributed to SMC3 acetylation at K105, K106.

\section{SMC3-K105, K106 acetylation is not essential for SCC and cellular proliferation}

Because ESCO1 is key in mediating acetylation at K105 and K106 residues on SMC3 (Fig. 1D; Zhang et al. 2008; Minamino et al. 2015) and because ESCO1 ${ }^{-1-1-}$ cells feature chromosome arm cohesion defects (Fig. 1E), we next asked whether the smc3-QQ mutation suppresses the SCC defects of esco1 mutant cells. We established esco1 smc3-QQ cell lines using a strategy similar to that mentioned above (see Fig. 4A) but starting from $\mathrm{ESCO}^{-1-1-}$ cells. We observed that the double-mutant esco1 smc3- 

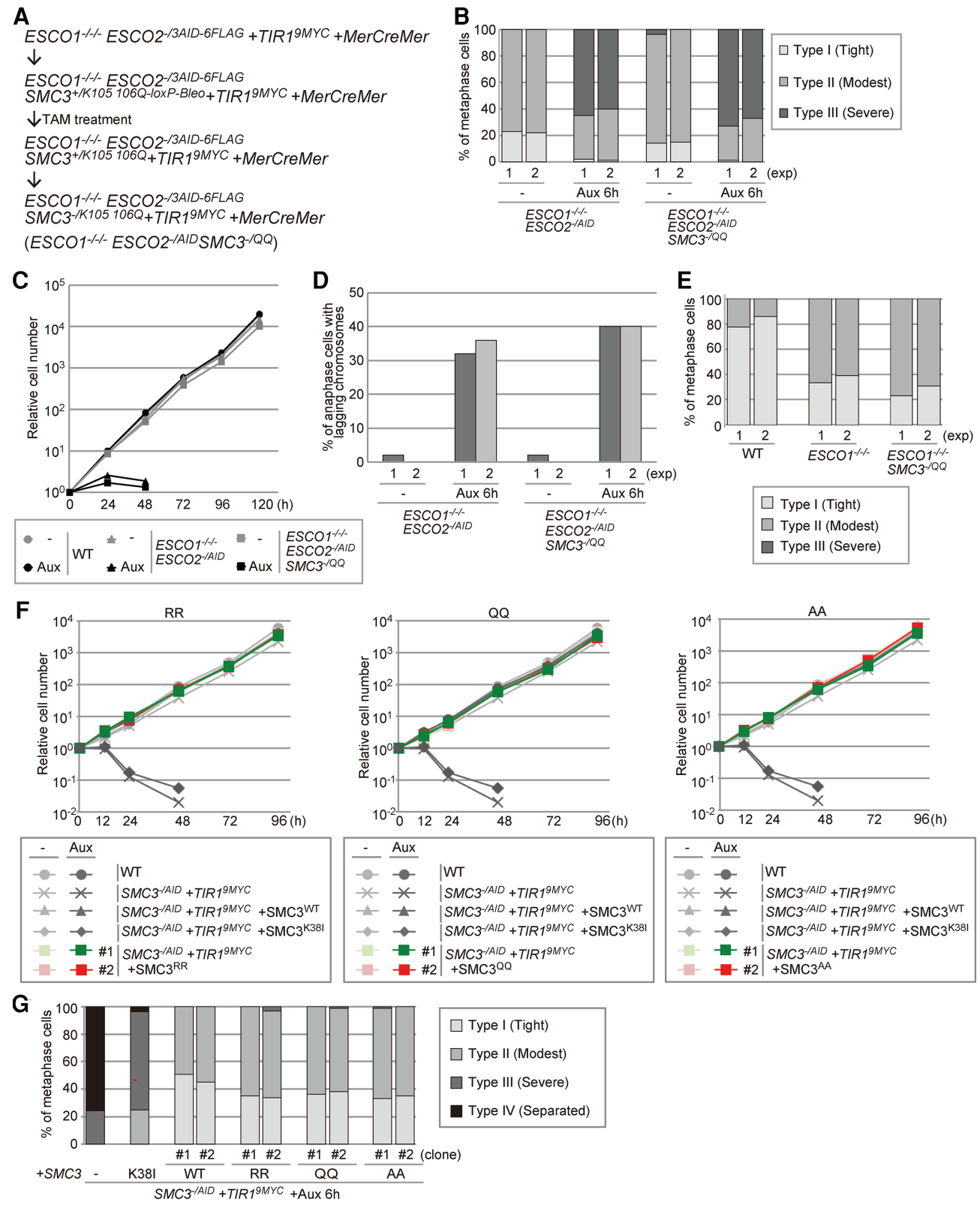

Figure 4. SMC3-K105, K106 acetylation is not singularly responsible for ESCO1's and ESCO2's roles in proliferation. (A) Scheme of the steps involved in establishing conditional ESCO1 $1^{-/-1-} E S C O 2^{-/ A I D} S M C 3^{-/ Q Q} .(B)$ Chromosomes from metaphase spreads were analyzed for cohesion defects as outlined in Figure 1E. Auxin $(500 \mu \mathrm{M}$ final) was added $6 \mathrm{~h}$ before sample collection. $(C)$ Growth curves are as in Figure 2C. Auxin was added at time 0, when indicated. $(D)$ The measurement of lagging chromosomes in anaphase cells was as in Figure 2E. Auxin (500 $\mu \mathrm{M}$ final) was added $6 \mathrm{~h}$ before cell collection. (E) Chromosomes from metaphase spreads were analyzed for cohesion defects as outlined in Figure 1E. $(F)$ Growth curves are as in Figure 2C. Auxin was added at time 0, when indicated. (G) Chromosomes from metaphase spreads were analyzed for cohesion defects as outlined in Figure 1E. Type IV indicates chromosomes with fully separated sister chromatids. Auxin was added $6 \mathrm{~h}$ before sample collection.

QQ had cohesion defects very similar to those of esco1 cells (Fig. 4E), indicating that SMC3 acetylation is not critical for the ESCO1 function in SCC.

Next, we decided to address the effects of mimicking acetylation or loss of acetylation at K105, K106 residues of SMC3 on proliferation and SCC when expressed as the sole sources of SMC3. To do so, we established SMC3-/3AID6Flag cells (see Supplemental Figs. S2A [for the SMC3 knockout construct], S3A,B [for SMC3-AID depletion's effect on cellular proliferation and depletion efficiency]). Next, we individually expressed in conditional SMC3-33AID6Flag cells different SMC3 variants-wildtype SMC3; ATPase-defective SMC3-K38I; acetylationdefective SMC3 variants K105R, K106R (RR) and SMC3 K105A, K106A (AA), and the acetylation-mimicking SMC3-QQ - as single sources of SMC3. After Auxin 
addition, SMC3-/3AID6Flag cells showed severe SCC and proliferation defects (Supplemental Fig. S3A) in the same range as RAD21-deficient cells (Sonoda et al. 2001), confirming that the SMC3-AID allele that we established is functional in the absence of Auxin and is efficiently inactivated by addition of Auxin. The SMC3 expression constructs randomly integrated in the genome had an HA tag for convenient detection and were expressed from a $\beta$-actin promoter. Importantly, exogenously expressed wild-type SMC3-HA-but not an ATPasedead mutant of SMC3 (SMC3-K38I) previously shown to be required for viability (Elbatsh et al. 2016) - compensated for the proliferation defects of Auxin-treated SMC3 ${ }^{-/ 3 A I D 6 F l a g}$ cells (Supplemental Fig. S3A). The overall levels of SMC3-K38I-HA were similar to the ones of wild-type SMC3-HA (Supplemental Fig. S3B).

Next, we compared the effects of expressing HA-tagged SMC3 wild-type, ATPase-dead K38I, acetylation-defective RR and AA, and acetyl-mimicking QQ variants in SMC3-/3AID6Flag cells. The expression levels of all of these variants were similar to the wild-type and K38I variants (Supplemental Fig. S3B). Notably, expression of QQ, RR, and AA variants restored the viability and SCC of Auxin-treated SMC3 ${ }^{-/ 3 A I D 6 F l a g}$ cells to the same degree as expression of the wild-type SMC3 construct (Fig. 4F,G). These results suggest that SMC3 acetylation at K105, $\mathrm{K} 106$ is not essential for proliferation in vertebrate cells (as both $s m c 3-R R$ and $s m c 3-A A$ cells are viable differently from $s m c 3-K 38 I$ ) or for SCC. Taken together with our previous results on the lack of effect of the smc3-QQ mutation in esco1 and esco1 esco2 cells, we conclude that ESCO1/2's essential functions either are manifested independently of SMC3 acetylation at K105, K106 or at the very least involve additional substrates besides the K105 and K106 sites of SMC3.

Cohesin release and sister chromatid separation in escol esco2 cells are suppressed by depletion of WAPL

Cohesin acetylation by ESCO1/2 is thought to promote SCC and proliferation by preventing WAPL-mediated removal of cohesin (Rowland et al. 2009; Sutani et al. 2009; Ladurner et al. 2016). With the newly established tools and cell lines, we sought to address the relationship between ESCO1/2-mediated events in cellular proliferation and WAPL function. Because WAPL knockout causes severe growth defects in mammalian cells (Tedeschi et al. 2013), we established a conditional wapl mutant. To these ends, we replaced one allele of WAPL with a knockout construct (Supplemental Fig. S4A) and tagged the second WAPL allele with a 3AID-6Flag tag in both wild-type and esco1 esco2 conditional cells expressing TIR1 (Fig. $5 \mathrm{~A})$. No growth defects were observed in conditional wapl and esco1 esco2 wapl cells without Auxin. After Auxin addition, WAPL-3AID-6Flag and ESCO2-3AID6Flag protein levels were strongly reduced (Fig. 5B). The metaphase chromosomes of wapl conditional cells revealed strongly cohered unseparated prometaphase-like chromosomes (Fig. 5C), in line with previous reports in other model systems (Gandhi et al. 2006; Tedeschi et al.
2013). This phenotype of strongly cohered unseparated chromosomes was also observed in conditional esco1 esco2 wapl cells, as expected (Fig. 5C).

The "unseparated" chromosome phenotype observed in the absence of WAPL most likely indicates persistent cohesin association to chromosomes (Tedeschi et al. 2013). To verify this, we immunostained cohesin (SMC3) in metaphase spreads. While cohesin was not visible in control cell lines $\left(W A P L^{-/ A I D}\right)$, WAPL depletion with Auxin caused SMC3 persistence along chromosomes in both wild-type and esco1 esco2 backgrounds (Fig. 5D). To address whether the unseparated chromosomes in WAPL-depleted cells are indeed caused by cohesin persistence, we next established SMC3-/3AID6Flag $W A P L^{-/ 3 A I D 6 \text { Flag }}$ cells. Six hours after addition of Auxin, both SMC3-3AID6Flag and WAPL-3AID-6Flag were degraded (Supplemental Fig. S4B). In these conditions, the "unseparated" chromosome phenotype of wapl mutants fully depended on cohesin (Supplemental Fig. S4C), in agreement with previous findings in MEFs (Tedeschi et al. 2013). Altogether, the results indicate that in the absence of WAPL, cohesin persists on metaphase chromosomes regardless of its acetylated state at K105, K106 and the ESCO1/2 function.

The proliferation and chromosome segregation defects of the escol esco2 double mutant are enhanced by WAPL depletion

We next addressed whether the persistence of cohesin on metaphase chromosomes caused by WAPL depletion rescues the lethality of the esco1 esco2 double mutant. Notably, the proliferation defect of esco1 esco2 cells was strongly exacerbated by coincident WAPL depletion and not rescued to the level of the wapl mutant, which displayed only mild growth defects (Fig. 5E). To gain more insight in the fast lethality of esco1 esco2 wapl cells, we assessed cell cycle distribution in these mutants versus control cell lines (Fig. 5F). We observed that esco1 esco2 wapl cells are largely in S phase $6 \mathrm{~h}$ after Auxin addition, similar to control wapl and esco1 esco2 cells. However, after completion of the S phase, esco1 esco2 wapl cells arrested in G2/M and did not cycle further (Fig. 5F), suggesting that problems arise during the first mitosis when WAPL and ESCO1/2 are concomitantly inactivated.

To examine this hypothesis and gain insight into the underlying molecular events, we assessed for chromosome missegregation defects in esco1 esco2 cells in the presence or absence of WAPL, the latter induced by $6 \mathrm{~h}$ of Auxin treatment (Fig. 5G). esco1 esco2 cells had an increase in lagging chromosomes (scored by the CENP-T dots present between the separating nuclear DNA bundles), a trend observed previously in other mutants suffering from increased centromeric sister chromatid separation (Figs. 2E, 5G; Abe et al. 2016). Lagging chromosomes were not observed in wapl cells, which were instead characterized by increased frequency in chromosome bridges (Fig. 5G), in line with previous reports (Haarhuis et al. 2013; Tedeschi et al. 2013). Notably, esco1 esco2 wapl cells had a new and different phenotype compared with esco1 esco2 and wapl mutants. 


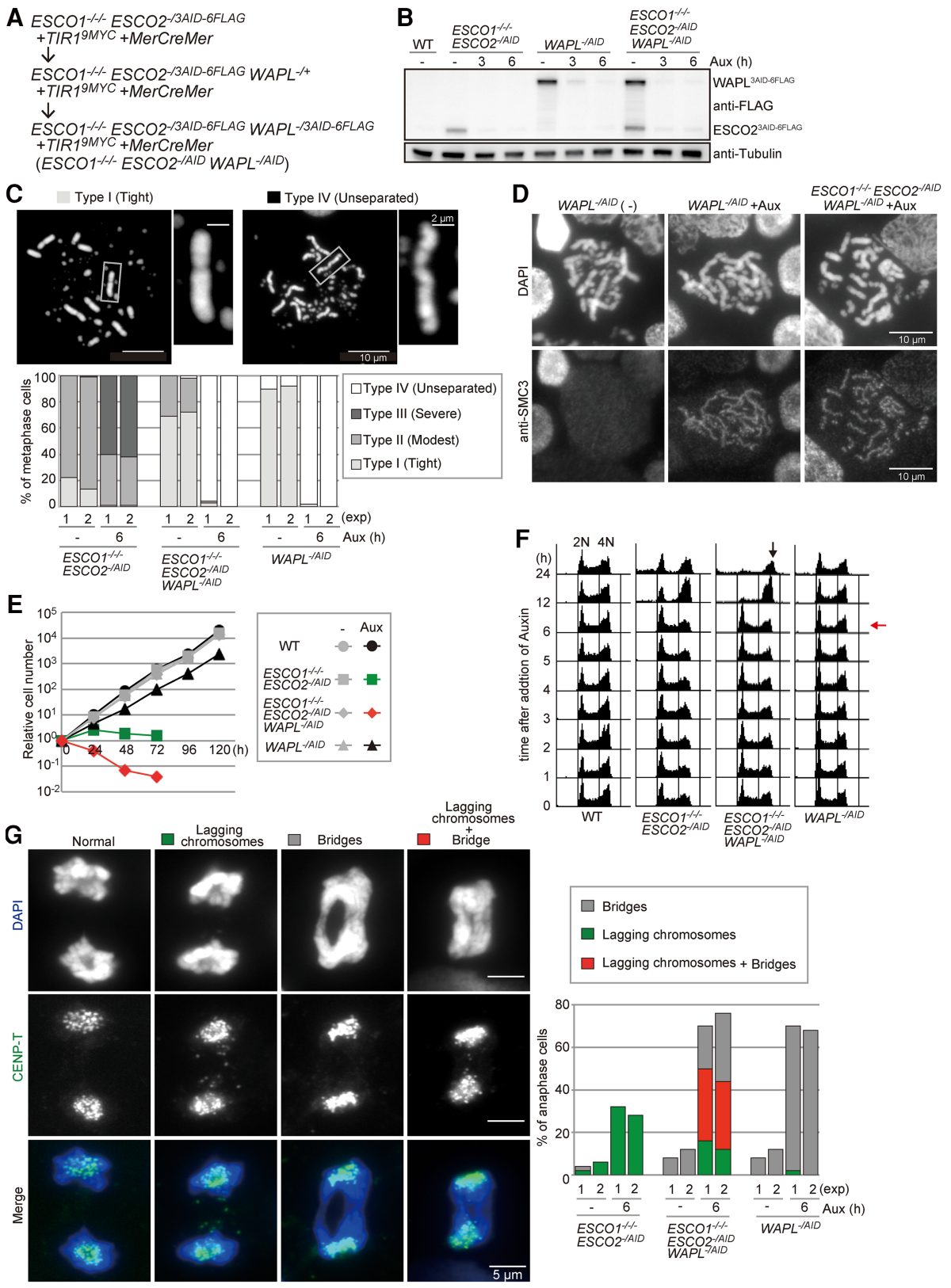

Figure 5. Cohesin release in esco1 esco2 cells is suppressed by depletion of WAPL but is associated with mitotic arrest and chromosome

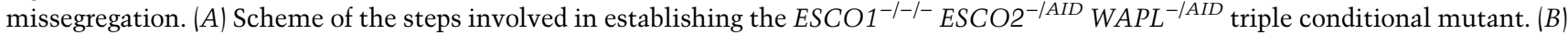
Depletion of ESCO2-3AID-6Flag protein and WAPL-3AID-6Flag protein was confirmed by Western blotting. Similar trends were observed in three independent experiments. $(C)$ Chromosomes from metaphase spreads were classified in four groups (see images for the new type IV [unseparated]) (Fig. 1E), and >100 metaphase cells were analyzed for each genotype. The results of two independent experiments are plotted. Auxin $(500 \mu \mathrm{M}$ final) was added $6 \mathrm{~h}$ before cell collection, when indicated. $(D)$ Immunostaining using the anti-Smc3 antibody and DAPI. After incubation with $100 \mathrm{ng} / \mathrm{mL}$ colcemid for $1 \mathrm{~h}$, metaphase spread samples were prepared by the cytospin method (Abe et al. 2016). (E) Growth curves are as in Figure 2C. $(F)$ Cell cycle progression for the indicated cell lines was analyzed by propidium iodide staining. Samples were taken at indicated time points. Auxin was added at time point $0 .(G)$ Chromosome bridges and missegregation in anaphase. At least 50 cells for the anaphase plot were analyzed for each experiment. The results of two independent experiments are plotted. The experiments were performed as in Figure 2E. Auxin (500 $\mu \mathrm{M}$ final) was added $6 \mathrm{~h}$ before cell collection.

Specifically, $20 \%$ of esco1 esco2 wapl anaphase cells were characterized by the concomitant presence of lagging chromosomes and chromosome bridges (Fig. 5G). We posit that the coincident presence of lagging chromo- somes and bridges in esco1 esco2 wapl cells leads to catastrophic mitosis and faster cell death than the occasionally induced aneuploidy caused by lagging chromosomes in esco1 esco2 cells. 
ESCO1/2 mediate the organization of interphase chromosome territories

WAPL is important for the organization of cohesin-defined chromosome territories, as observed in post-mitotic MEFs and haploid human cell lines mutated in WAPL (Tedeschi et al. 2013; Busslinger et al. 2017; Haarhuis et al. 2017). When we analyzed the pattern of cohesin staining in DT40 interphase cells $16 \mathrm{~h}$ after WAPL depletion) (see Fig. 5F), we did not observe very strong compaction--described previously as "vermicelli"-in postmitotic MEFs lacking WAPL (Tedeschi et al. 2013), although increased granularity was evident (Fig. 6A). To address whether this may be caused by the incomplete depletion of WAPL achieved with our WAPL-AID system, we established a Tet-off-WAPL construct (Supplemental Fig. S5A). WAPL shutoff cells were still viable, likely permitted by the p53-deficient status of DT40 cells (Abe and Branzei 2014), but did not lead to a typical "vermicelli" phenotype (Supplemental Fig. S5B) as initially described in post-mitotic MEFs. Recently, U2OS cells deficient in WAPL were also reported to lack a clear vermicelli phenotype (Rhodes et al. 2017). These differences are likely caused at least in part by the fact that DT40 and U2OS cells are dividing cells, and cohesin spends less time on chromatin before its removal by separase. We conclude that distinct model systems lead to differently appearing chromosome territories, the morphology of which may also vary based on the cohesin subunit that is analyzed (SMC3 here vs. SCC1 in previous studies) (Tedeschi et al. 2013; Busslinger et al. 2017; Haarhuis et al. 2017).

Considering the additive effects of wapl and esco1/2 mutations on proliferation (Fig. 5E), we next examined the appearance of interphase chromosome territories revealed by SMC3 staining in esco1 esco2 wapl cells versus control cell lines. We found an increased cohesin signal with a granular aspect in wapl mutants and a reduced, diffused signal for cohesin in esco1 esco2 cells as compared with $W A P L^{-/ A I D}$ control cells (Fig. 6A). Strikingly, a different pattern was observed in esco1 esco2 wapl cells in which the SMC3 staining resembled a brain-like pattern (Fig. 6A; Supplemental Fig. S5B, note similar trends in multiple nuclei). To address whether the cohesin staining pattern is relevant for DNA/chromatin territories, we calculated the maxima and minima of DNA and cohesin intensities across singular nuclei for wapl and esco1 eso2 wapl cells (Fig. 6B). In both cases, there was a general match, with nearly complete overlap between DNA and cohesin intensities in esco1 esco2 wapl cells, thus corroborating the notion that cohesin staining is a good readout for interphase chromatin territories.

To have a better view of the differences among various cell lines, we also used superresolution stimulated emission depletion (STED) microscopy (Fig. 6C). The results confirmed our previous observation that simultaneous loss of ESCO1 and ESCO2 affects interphase chromatin organization in wapl mutants, causing a different pattern of cohesin staining on chromatin. The altered interphase chromosome territories are likely to be associated with a different pattern of gene expression. To address this, we analyzed by RNA sequencing (RNA-seq) the gene expression pattern in wild-type, esco1 esco2, wapl, and esco1 esco2 wapl after treatment of cells with Auxin for $6 \mathrm{~h}$. Marked differences were found between the different genotypes (Supplemental Fig. S6A). These data reveal important roles for both ESCO1/2 and WAPL in gene expression regulation (Supplemental Fig. S6A-C). Taken together, these results indicate that ESCO1/2-mediated events contribute to the formation/architecture of interphase chromosome territories, which subsequently affect gene expression.

\section{ESCO1/2 negatively regulates cohesin association to interphase chromatin}

We next asked whether the organization of interphase chromosome territories is influenced by the amount of cohesin bound to chromatin and/or its binding state. Different pools of cohesin binding have been described, with cohesin bound in G1 being more loosely associated with chromatin, whereas the acetylated cohesin was bound longer to chromatin and was resistant to removal by WAPL (Ladurner et al. 2016; Rhodes et al. 2017). We performed chromatin fractionation experiments using synchronously growing cells treated with Auxin for $6 \mathrm{~h}$ and enriched in interphase (see Fig. 5F). These experiments revealed that more cohesin is associated with interphase chromatin in esco1 esco2 wapl cells compared with wild-type, esco1 esco2, and wapl cells, but there were no statistically significant differences between wapl and esco1 esco2 wapl cells in regard to the overall amount of bound cohesin (Supplemental Fig. S7A). As expected, the Ac-Smc3 levels were strongly reduced in esco1 esco2 and esco 1 esco 2 wapl cells (Supplemental Fig. S7B). Moreover, in wapl cells, the amount of Ac-Smc3 versus total Smc3 on chromatin was strongly increased (Supplemental Fig. S7C), in line with previously proposed models of cohesin removal (Ladurner et al. 2016).

Next, we immunostained cohesin in interphase nuclei in the absence or presence of nuclear membrane-permeabilizing treatment with detergent (Fig. 7). In the absence of nuclear membrane permeabilization, both nucleoplasmic cohesin and chromatin-bound cohesin were visualized, whereas nuclear permeabilization with Triton X100 led to the washout of the free nucleoplasmic cohesin pool, allowing one to observe the differences in the chromatin binding of cohesin in the different backgrounds analyzed. Using the first procedure, no differences in the normalized SMC3 intensities were observed among different genotypes and between wapl and esco1 esco2 wapl (Fig. 7A-C). On the other hand, for the second approach, a statistically significant increase in cohesin intensity was observed for wapl and esco1 esco2 wapl compared with wild-type and esco1/2 and, importantly, in esco1 esco2 wapl compared with wapl (Fig. 7D-F), following the trend already observed in chromatin fractionation studies (Supplemental Fig. S7A). As SMC3 acetylation is defective in esco1 esco2 wapl cells (differently from wapl) (Supplemental Fig. S7B) but the overall intensity of chromatin-associated cohesin was increased 
A

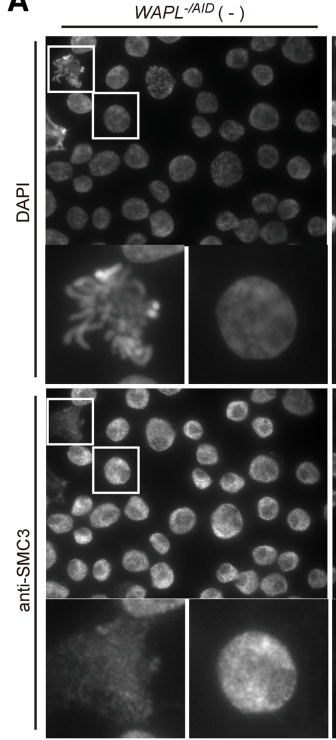

ESCO1 $1-\mathrm{ESCO}^{-/ A I D+A u x} 6 \mathrm{~h}$

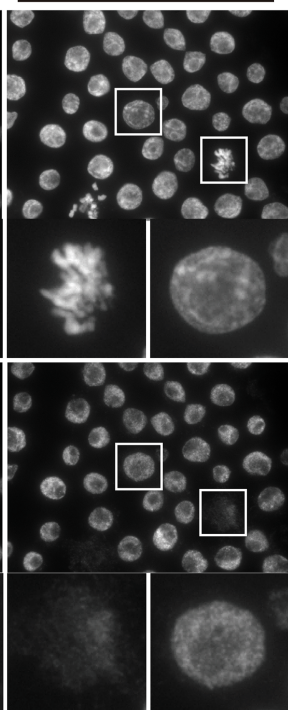

$W A P L^{-A A D}+A u x 6 h$

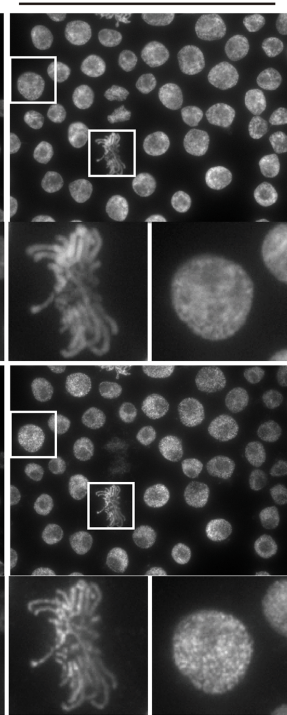

ESCO1 1 - - ESCO $-1 / A 1 D$

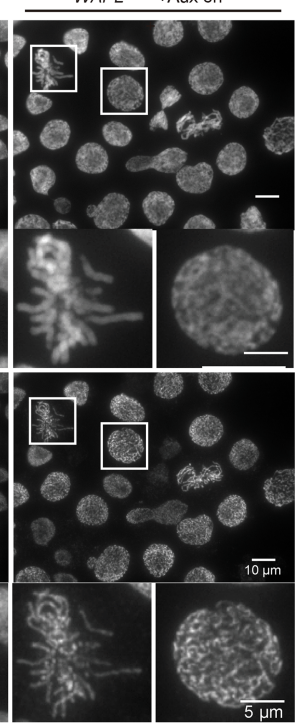

B

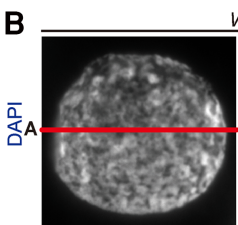

WAPL-AIID +Aux $6 \mathrm{~h}$

ESCO1- - ESCO2 $^{-/ A I D}$ WAPL-/AID +Aux $6 \mathrm{~h}$
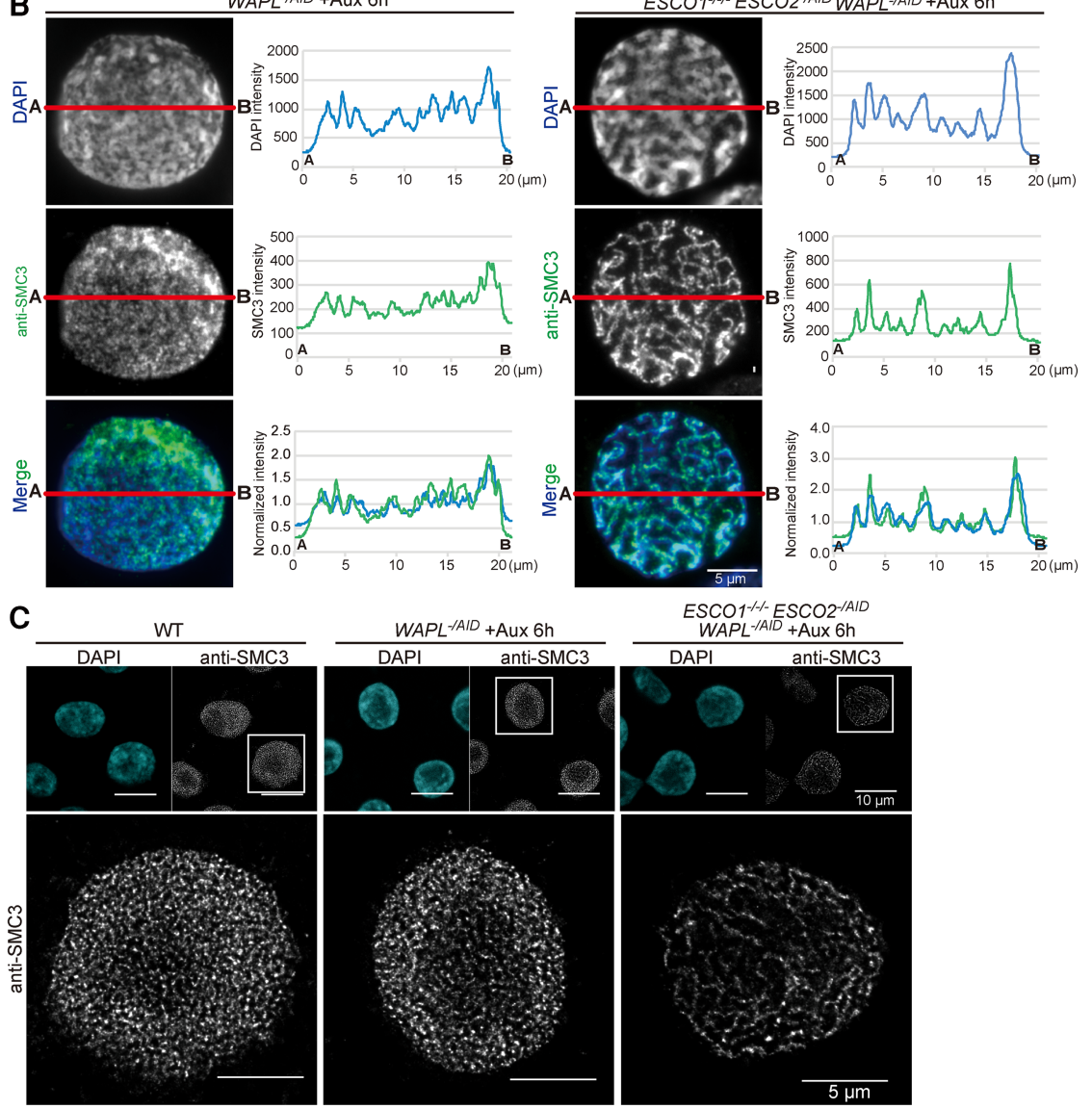

Figure 6. ESCO1/2-mediated events are implicated in the organization of interphase chromosome territories. $(A) \operatorname{Immunostaining~using~}$ the anti-SMC3 antibody and DAPI. Samples were prepared by the cytospin method. After the centrifugation, cells were fixed with $4 \%$ PFA in PBS containing $0.1 \%$ Triton X-100. Auxin ( $500 \mu \mathrm{M}$ final) was added $6 \mathrm{~h}$ before sample collection. (B) DAPI and immunostaining with the anti-SMC3 antibody following nuclear membrane permeabilization. Samples were prepared by the cytospin method. Before the centrifugation, cells were treated with $0.3 \%$ Triton X-100 for $5 \mathrm{~min}$. Auxin (500 $\mu \mathrm{M}$ final) was added $6 \mathrm{~h}$ before sample collection. The signal intensities versus distance along the indicated line, A to B, were plotted using ImageJ's analytical functions. For merged graphs, each signal intensity was normalized to the mean, and normalized intensities versus micrometer were plotted. Auxin (500 $\mu \mathrm{M}$ final) was added $6 \mathrm{~h}$ before sample collection. (C) Immunostaining using an anti-SMC3 antibody and DAPI. Images were taken by stimulated emission depletion (STED) microscopy. Samples were prepared by the cytospin method as in A. Auxin (500 $\mathrm{MM}$ final) was added $6 \mathrm{~h}$ before cell collection. 
A

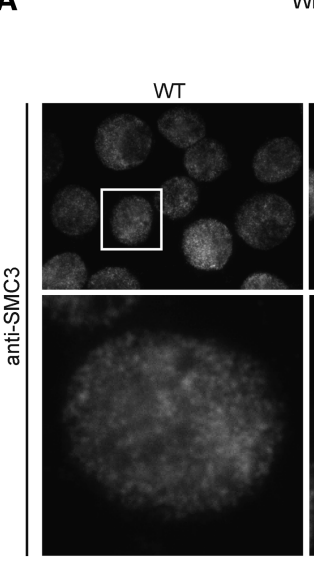

B

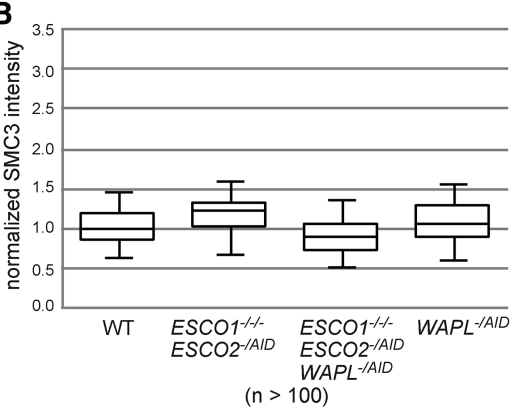

ESCO $1^{-1 /}$ Without nuclear membrane
permeabilization $\rightarrow$ Fixation with 4\% PFA
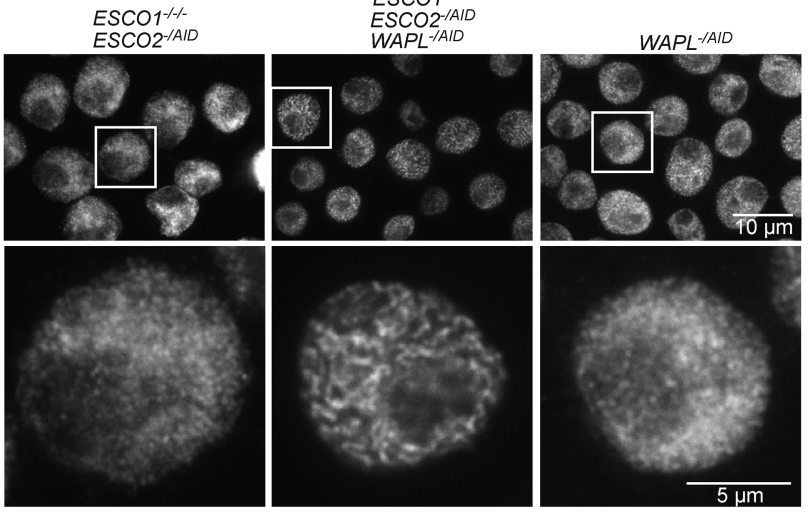

C

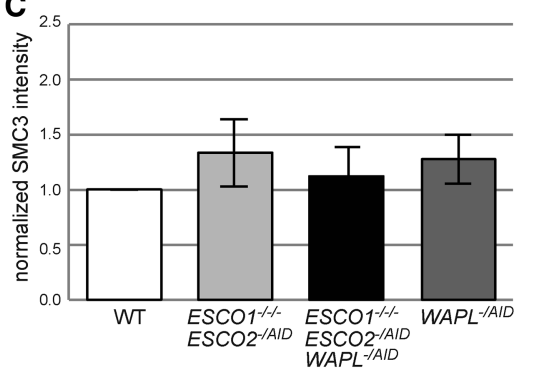

Figure 7. Cohesin binding to interphase chromatin is negatively regulated by ESCO1/2. Immunostaining using the antiSMC3 antibody and DAPI without $(A)$ or following $(D)$ nuclear membrane permeabilization to allow visualization of cohesin in nuclei $(A)$ or cohesin bound on chromatin $(D)$. Auxin (500 $\mu \mathrm{M}$ final) was added $6 \mathrm{~h}$ before sample collection. $(B, E)$ The nucleic area was defined by the DAPI signal. The SMC3 signals that overlapped with DAPI signals were measured and normalized to the SMC3 signal intensity in wild type and then plotted for individual representative experiments in which $>100$ nuclei were examined for each condition. (Middle line) Median; (box) 25th and 75th percentiles; (bars) 5th and 95th percentiles. $(C, F)$ The median values of three independent experiments were averaged, and plotted. $P$-values were calculated by Student's $t$-test. $\left(^{*}\right) P<$ $\left.0.05 ;{ }^{* *}\right) P<0.01$.

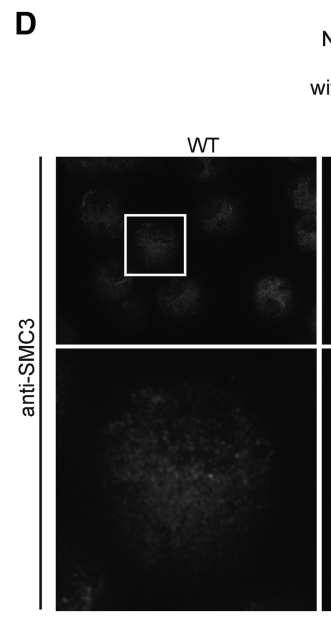

E

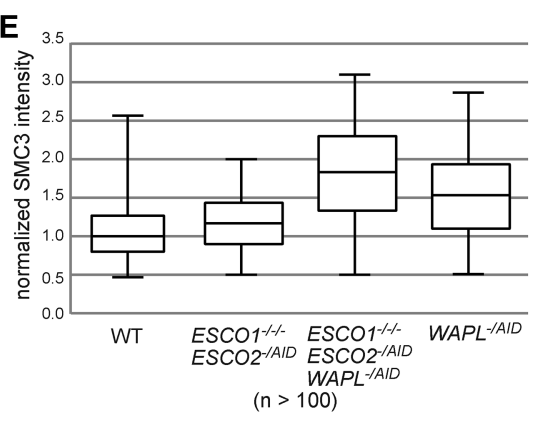

in esco1 esco2 wapl cells versus wapl (Fig. 7D-F), the results indicate that ESCO1/2 negatively regulates cohesin association with chromatin in interphase, working in the same direction as WAPL in this regard. This unexpected result may explain in part the synthetic phenotype between ESCO1/2 and WAPL depletion in regard to proliferation and opens the path for research to understand ESCO1/2's functions in negatively regulating cohesin 
binding and allowing proper chromatin territory organization in interphase.

\section{Discussion}

ESCO1 and ESCO2 acetyltransferases are required for genome integrity and human development. Both ESCO1 and ESCO2 can acetylate SMC3 at evolutionarily conserved sites, but the common and differential functions of ESCO1/2 in different chromosome metabolism settings and the contribution of SMC3 acetylation remain largely unknown. Using conditional depletion of factors and knock-in/knockout of genes, we established DT40 cell lines with multiple conditional knockout and point mutations in cohesin-related genes and regulators. With these tools at hand, we uncovered a hitherto unknown role for ESCO1/ESCO2 in interphase chromatin structure organization and revealed functional interaction and separationof-function roles for ESCO1 and ESCO2 in centromeric integrity and somatic cell proliferation.

Notably, we identified key roles for ESCO2 in supporting centromeric sister chromatid proximity and proliferation, with these functions being at least in part uncoupled from its roles in supporting chromosome arm SCC. The function of ESCO2 in centromere integrity and proliferation can be fully overtaken by high levels of ESCO1, whereas the chromosome arm SCC defects of esco2 are only in part suppressed by ESCO1 overexpression. Regarding to what extent SMC3 acetylation, which is induced by ESCO1 overexpression, is responsible for the above-mentioned events, we found that the acetylmimicking smc3-QQ mutation did not rescue the lethality and SCC defects of esco1 esco2 cells or the chromosome arm SCC defects of esco1 cells. Moreover, SMC3 acetylation was dispensable for the ability of cells conditionally depleted for cohesin (SMC3) to proliferate and maintain centromere proximity. These results indicate that SMC3 acetylation at K105, K106 is not singularly responsible for ESCO $1 / 2$ 's essential roles in proliferation and centromere integrity.

An intriguing question is why organisms increased the number of cohesin acetyltransferases during evolution. It was reported that the protein level of ESCO1 is constant during the cell cycle, while the expression of ESCO2 peaks up in S phase when SCC is established (Hou and Zou 2005). Moreover, ESCO1-mediated acetylation of SMC3 depends on PDS5 and is independent of DNA replication (Minamino et al. 2015). It is possible that cells need more ESCO1/2 acetyltransferase activity in S/G2 phase to establish and maintain cohesion and promote sister chromatidmediated recombination repair, especially in late replicating or difficult to replicate regions such as centromeres, which appear to be very sensitive to replication stress (Abe et al. 2016). As repetitive regions are more abundant in vertebrate genomes, we posit that multiple cohesin acetyltransferases evolved to optimally handle DNA metabolism processes associated with the replication of these loci.

It is important to understand whether the essential functions of ESCO1/2 are related to regulation of SCC or regulation of other chromosomal structural processes relying on cohesin. In DT40 cells, the smc3-QQ mutation did not suppress the lethality, chromosome segregation, and centromeric separation defects of esco 1 esco 2 cells or the chromosome arm cohesion defects of esco1. We reasoned that ESCO1/2 might be essential to stabilize cohesin on chromosomes, for instance, by preventing its removal by WAPL (Rudra and Skibbens 2013). The rescue of $e c o 1 \Delta$ lethality by $w p 11 \Delta$ in budding yeast supports this scenario (Rolef Ben-Shahar et al. 2008; Rowland et al. 2009). If the same were true in vertebrate cells, WAPL depletion-which, in DT40 cells, causes only a mild proliferation defect-should stabilize cohesin on chromosomes and suppress the lethality of esco1 esco2 cells. We found the former proposition to be true but not the latter.

Conversely from the initial prediction, the proliferation defect of conditional esco1 esco2 cells was strongly exacerbated by WAPL depletion, and the triple mutant esco1 esco2 wapl had severe chromosome segregation defects characterized by the unusual coincidence of chromosome bridges and lagging chromosomes, which may explain the fast lethality in these cells. Remarkably, in esco1 esco2 wapl mutants, the cohesin staining on interphase chromatin also revealed a different structure, similar, at a glance, to the one reported by Tedeschi et al. (2013) in G0-arrested MEFs or haploid human cells deleted for WAPL (Haarhuis et al. 2017). Notably, this phenotype is not observed in equivalent single wapl mutants in interphase DT40 cells or in U2OS cells mutated for WAPL (Rhodes et al. 2017). We suspect that these differences are intrinsic to the chromatin organization features and cohesin dynamics in proliferating versus G0-arrested cell lines and may also be influenced by the cohesin subunit detected and/or differences in the expression levels of ESCO1/2 in different systems or cell cycle phases (Tedeschi et al. 2013; Haarhuis et al. 2017). Although the exact reasons behind these chromatin architectural divergences are not known, the DT40 system used here allowed us to uncover a role for ESCO1/2 in the organization of interphase chromatin territories. Not surprisingly, we observed profound differences in gene expression upon ESCO1/2 and WAPL depletion.

Exploring the mechanism and substrates of ESCO1/2 that regulate the binding of cohesin to chromatin and the shaping of chromatin territories in interphase is an exciting undertaking for future studies of chromosome structure regulation.

\section{Material and methods}

The cell lines are listed in Supplemental Table S1. mRNA isolation, RT-PCR, Western blotting, chromosome analysis, immunofluorescence analysis, and cell cycle analysis were performed as described (Abe et al. 2016). Plasmid construction and transfection are described in the Supplemental Material.

Immunofluorescence following nuclear membrane permeabilization

Cells were transferred to microtubes and incubated for $3 \mathrm{~min}$ on ice. Triton X-100 was added to a final concentration of $0.3 \%$, 
incubated for 5 min on ice, and then spun onto slides with a cytocentrifuge. This protocol was adapted from Neelsen et al. (2013). The subsequent procedures were performed as described (Abe et al. 2016).

Immunofluorescent images by superresolution microscopy (STED)

Cells were spun onto slides with a cytocentrifuge and fixed by $4 \%$ paraformaldehyde in PBS for $10 \mathrm{~min}$ at room temperature. Fixed cells were then permeabilized by $0.5 \%$ Triton in PBS for $10 \mathrm{~min}$ at room temperature, rinsed with $0.5 \%$ BSA, and incubated for $1 \mathrm{~h}$ at room temperature with the anti-SMC3 antibody (a gift from Dr. Ana Losada, Spanish National Cancer Research Centre [CNIO]) (Losada et al. 1998). Binding of primary antibody was then detected with Atto674-conjugated goat anti-rabbit IgG (Sigma) at an appropriate concentration in PBS/0.5\% BSA. Chromosomes and nuclei were counterstained with DAPI at $0.2 \mu \mathrm{g} /$ $\mathrm{mL}$. Immunofluorescence images were collected with a $100 \times$ NA 1.40 objective lens together with a filter wheel by SP8 confocal microscopy equipped with STED (Leica) at room temperature. Subsequent analysis and processing of images were performed using LAS-X software (Leica) and Image (National Institutes of Health). All images were scaled and processed identically. Immunofluorescence images were collected on a Leica TCS SP8 STED $3 \times$ superresolution microscope using the HC PL APO 100×/1.40 oil immersion objective. After acquisition, the images were deconvolved with SVI Huygens Professional software.

\section{Chromatin-binding assay}

The chromatin-binding assay was performed using the subcellular protein fractionation kit for cultured cells (Thermo) according to the manufacturer's protocol.

\section{Ion torrent library preparation and sequencing}

One microgram of total RNA was polyA-selected using the Dynabeads mRNA direct micropurification kit (Thermo) according to manufacturer's protocol. After polyA selection, RNA were used to prepare strand-specific barcoded RNA libraries with Ion Total RNA-seq kit version 2.0 (Thermo) following manufacturer's protocol. The library qualities were checked by running on a BioAnalyzer HS DNA 2100, and the concentrations were determined from the analysis profiles. Two bar-coded libraries were pooled together on an equimolar basis and run using three PIv3 chips on an Ion Torrent Proton using HiQ200 chemistry.

\section{RNA-seq data bioinformatics analysis}

Sequence reads were demultiplexed and processed for adapter trimming by Ion Proton. These sequences were analyzed using the CLC Genomics Workbench (Qiagen) as described previously (Arakawa 2016). Briefly, the sequence reads were aligned with the Ensemble chicken genome database (International Chicken Genome Sequencing Consortium 2004) using the RNA-seq analysis toolbox with the alignment parameters of a length fraction of $80 \%$ and a similarity fraction of $80 \%$. Expression levels of each gene were normalized by reads per kilobase of exon model per million mapped reads (RPKM). The differentially expressed genes between the different mutants were hierarchically clustered and visualized as a heat map. The numbers of genes identified under the parameters of a fold change of $\geq 1.5$ and a false discovery rate (FDR) $P$-value of $\leq 5 \%$ are displayed in Venn diagrams.

\section{Data quantification and analysis}

Signal intensities obtained in fluorescent microscopy experiments were quantified using ImageJ. DAPI signal was used to select nuclei and measure the fluorescence intensity of target proteins inside the nuclei. Quantifications were processed with Microsoft Excel.

\section{Acknowledgments}

We thank D. Parazzoli and E. Martini at the Imaging facility at the FIRC (Italian Foundation for Cancer Research) Institute of Molecular Oncology (IFOM) for help and advice on STED; S. Minardi and M. Riboni at Cogentech for RNA-seq library preparation and sequencing; A. Losada, K. Shirahige, and T. Fukagawa for reagents; C. Morrison, B. Szakal, and I. Psakhye for scientific discussions; and B. Szakal for designing the cover art. This work was supported by Fondazione Telethon (GGP12160), the Italian Association for Cancer Research (AIRC IG 14171 and IG 18976), and the European Research Council (Starting Grant 242928 and Consolidator Grant 682190) grants to D.B. T.A. was partly supported by the Structured International Post-Doc Program (SIPOD) fellowship cofunded in the context of the FP7 Marie Curie Actions-People.

\section{References}

Abe T, Branzei D. 2014. High levels of BRC4 induced by a Tet-On 3G system suppress DNA repair and impair cell proliferation in vertebrate cells. DNA Repair 22: 153-164.

Abe T, Kawasumi R, Arakawa H, Hori T, Shirahige K, Losada A, Fukagawa T, Branzei D. 2016. Chromatin determinants of the inner-centromere rely on replication factors with functions that impart cohesion. Oncotarget 7: 67934-67947.

Arakawa H. 2016. A method to convert mRNA into a gRNA library for CRISPR/Cas9 editing of any organism. Sci Adv 2: e1600699.

Borges V, Smith DI, Whitehouse I, Uhlmann F. 2013. An Eco1-independent sister chromatid cohesion establishment pathway in S. cerevisiae. Chromosoma 122: 121-134.

Busslinger GA, Stocsits RR, van der Lelij P, Axelsson E, Tedeschi A, Galjart N, Peters JM. 2017. Cohesin is positioned in mammalian genomes by transcription, CTCF and Wapl. Nature 544: 503-507.

Elbatsh AM, Haarhuis JH, Petela N, Chapard C, Fish A, Celie PH, Stadnik M, Ristic D, Wyman C, Medema RH, et al. 2016. Cohesin releases DNA through asymmetric ATPase-driven ring opening. Mol Cell 61: 575-588.

Era S, Abe T, Arakawa H, Kobayashi S, Szakal B, Yoshikawa Y, Motegi A, Takeda S, Branzei D. 2012. The SUMO protease SENP1 is required for cohesion maintenance and mitotic arrest following spindle poison treatment. Biochem Biophys Res Commun 426: 310-316.

Gandhi R, Gillespie PJ, Hirano T. 2006. Human Wapl is a cohesinbinding protein that promotes sister-chromatid resolution in mitotic prophase. Curr Biol 16: 2406-2417.

Guacci V, Koshland D. 2012. Cohesin-independent segregation of sister chromatids in budding yeast. Mol Biol Cell 23: 729-739.

Haarhuis JH, Elbatsh AM, van den Broek B, Camps D, Erkan H, Jalink K, Medema RH, Rowland BD. 2013. WAPL-mediated removal of cohesin protects against segregation errors and aneuploidy. Curr Biol 23: 2071-2077.

Haarhuis JHI, van der Weide RH, Blomen VA, Yanez-Cuna JO, Amendola M, van Ruiten MS, Krijger PHL, Teunissen $\mathrm{H}$, Medema RH, van Steensel B, et al. 2017. The cohesin release 
factor WAPL restricts chromatin loop extension. Cell 169: 693-707.e14.

Hou F, Zou H. 2005. Two human orthologues of Ecol/Ctf7 acetyltransferases are both required for proper sister-chromatid cohesion. Mol Biol Cell 16: 3908-3918.

International Chicken Genome Sequencing Consortium. 2004. Sequence and comparative analysis of the chicken genome provide unique perspectives on vertebrate evolution. Nature 432: 695-716.

Ivanov D, Schleiffer A, Eisenhaber F, Mechtler K, Haering CH, Nasmyth K. 2002. Ecol is a novel acetyltransferase that can acetylate proteins involved in cohesion. Curr Biol 12: 323-328.

Kobayashi K, Fujii T, Asada R, Ooka M, Hirota K. 2015. Development of a targeted flip-in system in avian DT40 cells. PLOS One 10: $\mathrm{e} 0122006$.

Ladurner R, Kreidl E, Ivanov MP, Ekker H, Idarraga-Amado MH, Busslinger GA, Wutz G, Cisneros DA, Peters JM. 2016. Sororin actively maintains sister chromatid cohesion. EMBO J 35: 635-653.

Losada A, Hirano M, Hirano T. 1998. Identification of Xenopus SMC protein complexes required for sister chromatid cohesion. Genes Dev 12: 1986-1997.

Luedeke M, Linnert CM, Hofer MD, Surowy HM, Rinckleb AE, Hoegel J, Kuefer R, Rubin MA, Vogel W, Maier C. 2009. Predisposition for TMPRSS2-ERG fusion in prostate cancer by variants in DNA repair genes. Cancer Epidemiol Biomarkers Prev 18: 3030-3035.

Minamino $M$, Ishibashi $M$, Nakato R, Akiyama $\mathrm{K}$, Tanaka $\mathrm{H}$, Kato Y, Negishi L, Hirota T, Sutani T, Bando M, et al. 2015. Escol acetylates cohesin via a mechanism different from that of Esco2. Curr Biol 25: 1694-1706.

Neelsen KJ, Zanini IM, Mijic S, Herrador R, Zellweger R, Ray Chaudhuri A, Creavin KD, Blow JJ, Lopes M. 2013. Deregulated origin licensing leads to chromosomal breaks by rereplication of a gapped DNA template. Genes Dev 27: 2537-2542.

Nishimura K, Fukagawa T, Takisawa H, Kakimoto T, Kanemaki M. 2009. An auxin-based degron system for the rapid depletion of proteins in nonplant cells. Nat Methods 6: 917-922.

Onn I, Guacci V, Koshland DE. 2009. The zinc finger of Ecol enhances its acetyltransferase activity during sister chromatid cohesion. Nucleic Acids Res 37: 6126-6134.

Peters JM, Bhaskara V. 2009. Cohesin acetylation: from antiestablishment to establishment. Mol Cell 34: 1-2.

Peters JM, Nishiyama T. 2012. Sister chromatid cohesion. Cold Spring Harb Perspect Biol 4: a011130.

Price JC, Pollock LM, Rudd ML, Fogoros SK, Mohamed H, Hanigan CL, Le Gallo M, Program NIHISCCS, Zhang S, Cruz P, et al. 2014. Sequencing of candidate chromosome instability genes in endometrial cancers reveals somatic mutations in ESCO1, CHTF18, and MRE11A. PLoS One 8: e63313.

Rahman S, Jones MJ, Jallepalli PV. 2015. Cohesin recruits the Escol acetyltransferase genome wide to repress transcription and promote cohesion in somatic cells. Proc Natl Acad Sci 112: $11270-11275$.
Rhodes JDP, Haarhuis JHI, Grimm JB, Rowland BD, Lavis LD, Nasmyth KA. 2017. Cohesin can remain associated with chromosomes during DNA replication. Cell Rep 20: 2749-2755.

Rolef Ben-Shahar T, Heeger S, Lehane C, East P, Flynn H, Skehel M, Uhlmann F. 2008. Ecol-dependent cohesin acetylation during establishment of sister chromatid cohesion. Science 321: 563-566.

Rowland BD, Roig MB, Nishino T, Kurze A, Uluocak P, Mishra A, Beckouet F, Underwood P, Metson J, Imre R, et al. 2009. Building sister chromatid cohesion: smc3 acetylation counteracts an antiestablishment activity. Mol Cell 33: 763-774.

Rudra S, Skibbens RV. 2013. Cohesin codes-interpreting chromatin architecture and the many facets of cohesin function. J Cell Sci 126: 31-41.

Skibbens RV, Corson LB, Koshland D, Hieter P. 1999. Ctf7p is essential for sister chromatid cohesion and links mitotic chromosome structure to the DNA replication machinery. Genes Dev 13: 307-319.

Sonoda E, Matsusaka T, Morrison C, Vagnarelli P, Hoshi O, Ushiki T, Nojima K, Fukagawa T, Waizenegger IC, Peters JM, et al. 2001. Scc1/Rad21/Mcd1 is required for sister chromatid cohesion and kinetochore function in vertebrate cells. Dev Cell 1: 759-770.

Stephan AK, Kliszczak M, Dodson H, Cooley C, Morrison CG. 2011. Roles of vertebrate Smc5 in sister chromatid cohesion and homologous recombinational repair. Mol Cell Biol 31: 1369-1381.

Sutani T, Kawaguchi T, Kanno R, Itoh T, Shirahige K. 2009. Budding yeast Wpl1(Rad61)-Pds5 complex counteracts sister chromatid cohesion-establishing reaction. Curr Biol 19: 492-497.

Tedeschi A, Wutz G, Huet S, Jaritz M, Wuensche A, Schirghuber E, Davidson IF, Tang W, Cisneros DA, Bhaskara V, et al. 2013. Wapl is an essential regulator of chromatin structure and chromosome segregation. Nature 501: 564-568.

Unal E, Heidinger-Pauli JM, Kim W, Guacci V, Onn I, Gygi SP, Koshland DE. 2008. A molecular determinant for the establishment of sister chromatid cohesion. Science 321: 566-569.

Vega H, Waisfisz Q, Gordillo M, Sakai N, Yanagihara I, Yamada M, van Gosliga D, Kayserili H, Xu C, Ozono K, et al. 2005. Roberts syndrome is caused by mutations in ESCO2, a human homolog of yeast ECO1 that is essential for the establishment of sister chromatid cohesion. Nat Genet 37: 468-470.

Whelan G, Kreidl E, Wutz G, Egner A, Peters JM, Eichele G. 2012. Cohesin acetyltransferase Esco2 is a cell viability factor and is required for cohesion in pericentric heterochromatin. EMBO J 31: 71-82.

Zhang J, Shi X, Li Y, Kim BJ, Jia J, Huang Z, Yang T, Fu X, Jung SY, Wang $Y$, et al. 2008. Acetylation of Smc3 by Ecol is required for $\mathrm{S}$ phase sister chromatid cohesion in both human and yeast. Mol Cell 31: 143-151.

Zhang S, Li J, Zhou G, Mu D, Yan J, Xing J, Yao Z, Sheng H, Li D, Lv C, et al. 2016. Increased expression of ESCO1 is correlated with poor patient survival and its role in human bladder cancer. Tumour Biol 37: 5165-5170. 


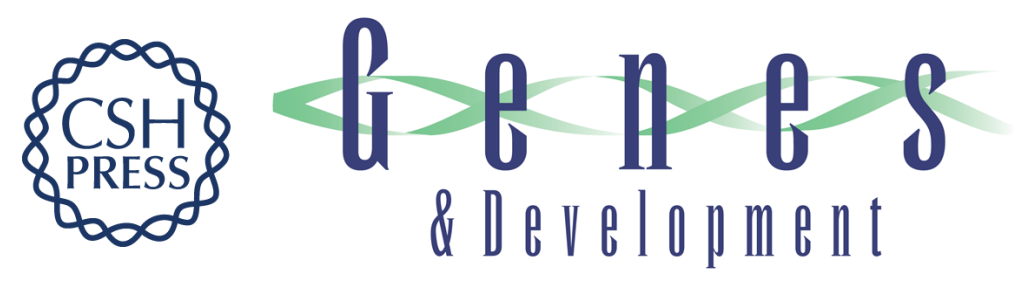

\section{ESC01/2's roles in chromosome structure and interphase chromatin organization}

Ryotaro Kawasumi, Takuya Abe, Hiroshi Arakawa, et al.

Genes Dev. 2017, 31: originally published online December 1, 2017

Access the most recent version at doi:10.1101/gad.306084.117

\section{Supplemental http://genesdev.cshlp.org/content/suppl/2017/12/01/gad.306084.117.DC1 \\ Material}

Related Content

References

Creative

Commons

License

Email Alerting

Service
Liberating cohesin from cohesion

Kerry Bloom

Genes Dev. November , 2017 31:2113-2114

This article cites 40 articles, 15 of which can be accessed free at:

http://genesdev.cshlp.org/content/31/21/2136.full.html\#ref-list-1

Articles cited in:

http://genesdev.cshlp.org/content/31/21/2136.full.html\#related-urls

This article, published in Genes \& Development, is available under a Creative Commons License (Attribution-NonCommercial 4.0 International), as described at

http://creativecommons.org/licenses/by-nc/4.0/.

Receive free email alerts when new articles cite this article - sign up in the box at the top right corner of the article or click here.

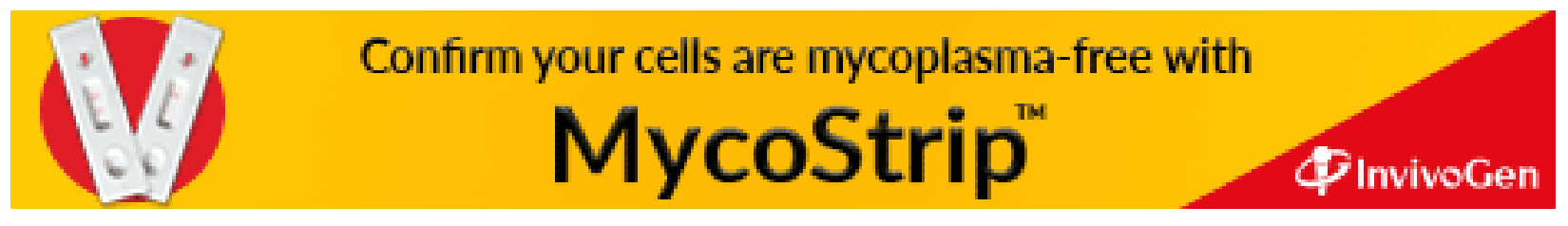

